\title{
Utilization of New Media among Farmers of Punjab: A Study of Moga District, India
}

\author{
Sheetal Thapar, Narinderpal Singh and Ranjeet Kaur* \\ Department of Agricultural Journalism, Languages and Culture, Punjab Agricultural \\ University, Ludhiana, India \\ *Corresponding author
}

\section{Keywords}

Online and mobile media, Agriculture, M-apps and social media

\section{Article Info}

Accepted:

17 September 2019

Available Online:

10 October 2019

\section{A B S T R A C T}

In the present era, new media has become a mainstay of information in the society which includes online and mobile media. Due to improved mobile technology, better internet connectivity and low cost of mobile phones and data plans, penetration of new media is increasing day by day in urban as well as rural India. It has also been providing the best opportunity to farmers to get new knowledge, ideas, information regarding agriculture like weather updates, marketing, crop insurance, government schemes etc. Keeping this in view, the present study is an endeavour to determine the availability and use of new media among farmers of Moga district and to identify the problems faced by them in accessing new media. A total of 180 farmers were randomly selected from the four villages of two blocks of Moga District for data collection by using the stratified random sampling. Out of this total, 45 farmers from each village (15 Marginal, 15 Small and 15 Large farmers) were randomly chosen and they were asked to fill the questionnaires. The results indicated that Majority of respondents had their own mobile phones while sixty per cent of them had availability of internet on their mobiles phones. However, half of the respondents got the weather updates through new media while a few of them were using mobile to make calls to extension experts and to establish link with markets. Language barrier is the most common problem faced by respondents and most of the respondents were unable to understand the technical words used in agricultural apps and websites. That is why, the study recommends that agricultural information available on apps and websites should be in local/vernacular language and technical jargons should be avoided. Furthermore, Cooperative societies should be roped in to create online and whatsapp groups to provide information to member farmers.

\section{Introduction}

India is an agrarian nation with around 70 per cent of its rural population directly and indirectly depending upon agriculture for their livelihood. Agriculture is the most important sector of Indian economy, though its contribution to the Gross Domestic Product (GDP) has decreased between 1990 to 1991 and 2010 to 2011 from $30.00 \%$ to $14.50 \%$ (Anonymous, 2012). In order to bring substantial development in the agricultural 
sector, there is need of reliable, timely and relevant information related to agriculture. Information is currently regarded as a factor of production just like labor, capital, and land (Rao, 2007). Use of information in agriculture sector is enhancing farming productivity in a number of ways. Relevant and timely information helps farmers' community to take right decision to sustain growth of agriculture activity. Providing information on weather trends, best practices in farming, timely access to market information helps farmer make correct decisions about what crops to plants and where to sell their produce and buy inputs.

Therefore, information is a powerful tool in addressing the agricultural needs and, if it is used properly, it could change nation's economics (Bachhav, 2012). Traditionally, newspapers, television and magazines have been the dominant media to disseminate agricultural information. In recent years, however, technology awareness, computer literacy and usage of smart phones and internet are increasing across all demographics in India.

Mobile phones and online media are now becoming the most suitable means to disseminate agriculture related information to farmers in rural areas. It has wide application and advantage which lead to its increasing use. Availability of cheap android phones has further added to increase in mobile internet users across geographical areas and socioeconomic groups. Mobile keeps people connected, helps to share and seek information. They are widely used for social networking, playing games, listening to music, sending e-mails, seeking information regarding health, education etc. and there is a significant rise in the use of mobile phones because of their potential for the quick transfer of information. Mobile communications technology has quickly become the world's most common way of transmitting voice, data, and services in the developing world.
Nowadays farmers are more aware about communication technology tools such as computer, internet and mobile phone and improving their standard of living (Yuen et al., 2003). Mobile phone technology for farmers has been started in developing countries and smart phone might be used to access e.g. personal bank account (Herzberg, 2003). The affordability and multifunctionality of smart phones are making mobile as the device of choice for users in both rural and urban India. But majority of the farmers still pertain to traditional agriculture system. For communication, farmers still prefer visiting agriculture stakeholders and do not use mobile phone for SMS and email. Most of mobile agriculture applications are not user-friendly and we should consider the illiteracy of farmers whenever developing such mobile application. The information such as agriculture, marketing, weather, advices should be local based and in local language. There are also educational and social barriers which need to be broken by academic institutions with due interest of government, NGOs, mobile phone companies and development participants. Farmers - a sizeable component of rural population, if well informed, can project the state into an even faster growth trajectory. The dream of an information-rich society can be realized by taking into account this major constituent of population for providing authentic information in an easily comprehensible format (Thapar and Nayal, 2018). Punjab can take maximum benefit by directly connecting with the consumers, buyers and experts with just the tap of the finger or click of a button. Connecting with the agriculturists of other communities, states or countries would open up new vistas where one can share ideas; improve productivity as well as standard of living. This could lift the standard and status of Punjab's agriculture even further.

Therefore, the study was carried out to understand the new media utilization pattern 
of farmers which would be helpful to formulate better ways and means to structure and shape content in the online and mobile media for them. It would lead to a more effective, efficient and productive use of media. This holds importance for the State in general and the country in particular as India aspires to become a prominent economic power in the near future. The objectives of the study were:

1. To study the demographic profile of farmers

2. To determine the availability and use of new media among farmers of Moga district

3. To identify the problems faced by farmers while accessing the new media.

\section{Materials and Methods}

The present study was exploratory in nature. It was conducted in rural areas of Moga district of Punjab. Stratified random sampling was used to select the respondents. At first level, out of total five blocks, Moga I and Dharamkot were the two blocks which were randomly selected for the present study. At second level, two villages were chosen from each block. Total four villages i.e. Maddoke and Mehna from Moga I and Bakarwala and Manawan from Dharmkot were chosen for the present study. At third level, 15 farmers each with equal representation of marginal $(<2$ acres), small ( $2 \geq$ and $<5$ acres) and large farmers ( $5 \geq$ acres) were randomly selected as respondents on the basis of their land holding. This made up 45 farmers from each village. Thus sample size was 180 farmers. They were asked to fill close-ended questionnaire in the language of their choice i.e. Punjabi and English. Data were collected regarding demographic profile viz age, education; land holdings, income etc., and availability and use of new media. The obtained data were tabulated and frequency distribution, percentages and averages were worked out for analysis of data.

\section{Results and Discussion}

The table 1 presents the demographic profile of respondents i.e. age, education, family type and annual income. Age of respondents varied between 20 to 60 years. Nearly two-third of them belonged to the age group of 40 years $\geq$ and $<60$ years and rest were above 60 years of age. Since the majority of respondents were of the age 40 years and above, it reflects on the current status of agriculture as occupation among villagers. With agriculture not remaining very remunerative, the youth is opting for other lines of employment. Nearly one-fourth of farmers $(23.33 \%, 42$ in number) belonged to young age group 20 years $\geq$ and $<$ 40 years. Out of these, 42.8 per cent were large farmers. It indicates that children of large farmers are more likely to adopt agriculture as a profession as it helps them to take care and maintain their property too.

In terms of educational qualifications, out of total respondents, 18.33 per cent were illiterate. Of this, 20 per cent and 21.67 per cent were marginal and small farmers. It is evident that illiteracy is more prevalent among marginal and large farmers. Overall, 31.67 per cent farmers were educated up to Matric, and 15.55 per cent had done senior secondary. Only 9.45 per cent farmers (17 in number) were graduate and above. There were only 1.67 per cent postgraduate ( 3 in number) and all of them were large farmers. Higher education was more common among large farmers due to better access to resources.

There are two types of families prevalent in Punjab i.e. nuclear family and joint family. The trend of nuclear families earlier found in urban areas has now made inroads in rural areas too. The results show that more than half of the respondents $(52.8 \%)$ were from joint 
families whereas 47.22 per cent belonged to nuclear families. The perusal of table 1 indicated that overall 27.22 per cent and 31.11 per cent of respondents had annual family income between Rs. $100000 \geq$ and $<300000$ and Rs. $300000 \geq$ and $<500000$ respectively whereas 18.33 per cent of them had income less than Rs. 100000 per annum. The results showed that majority of large farmers $(85 \%)$ had annual income of Rs. 300000 and above.

\section{Availability and Use of Mobile Media and Internet}

The possession of mobile phones and using internet, particularly, have become a necessity in the contemporary India irrespective of age, status, profession, income and place of residence. Mobile phones and internet have been regarded as widely accessed tools by farmers for communication and to get agriculture related information (Chhachar et al., 2014). Similar results are found in the present study. The perusal of table 2 indicated that majority (97.22\%) of farmers were having the accessibility and availability of mobile phones as it is affordable, portable as well as useful during emergencies while a few of them $(2.78 \%, 5$ in number) did not own the mobile phones. Out of these 5, 80 per cent were marginal farmers who did not have access to mobile phones due to illiteracy and poor economic conditions. More than onefourth $(27.78 \%)$ of farmers had one mobile at their home while nearly half $(48.33 \%)$ of them

and more than one-fifth $(22.22 \%)$ of respondents were having 'two' and 'three \& more' mobile phones at home, respectively. By comparing the three categories of farmers, it was found that five percent marginal farmers had no mobile at home. But small and large farmers were having mobile phones at their home. Regarding the type of mobile, it was found that 37.22 per cent and 40 per cent of farmers had 'only ordinary' and 'only smart phone' at home, respectively. Only 22.78 per cent of them were having the availability of both ordinary and smart phones at home. Half of marginal farmers had access to only ordinary mobile phones whereas 78.33 per cent of large farmers had smart phones. About 62.78 per cent respondents had internet connection on their phones. Category-wise, more than three-fourth of large farmers and 60 per cent of small farmers had internet facility on their mobile phones whereas half of marginal farmers had no availability of internet on their phones. Further, table 2 explains the average use of mobile phone in a day. It was seen that 43.89 per cent respondents were using mobile phone less than one hour. A closer look reveals that half of marginal and small farmers each were such low users of mobile phone.

Nearly half of respondents $(47.78 \%)$ were using mobile phones for one hour and above in a day. Only 8.33 per cent farmers did not use mobile phones out of which 15 per cent were marginal farmers whereas 8.3 per cent and 1.7 per cent were small and marginal farmers, respectively. The table points out the marked difference between average use of mobile phones between marginal and large farmers where marginal farmers were seen to be using them for lower number of hours as compared to the large farmers.

\section{Need of assistance by family members}

Despite 97.22 per cent penetration of mobile phones among the respondents, many could not make full use of the device due to lack of education, conservative attitude being less tech-savvy and lack of willingness to learn at an old age. In such cases, they took assistance of family members such as their children, nieces, nephews etc. in order to use the mobile phones to access information on them. Data presented in table 3 depicts that 48.89 per cent respondents were taking help of their family members whereas 51.11 per cent of 
respondents had no need of assistance from their family while operating online medium.

\section{Type of agricultural information searched through internet}

Farmers need various type of agricultural information in their daily life such as weather updates, marketing, input prices, new technology, new seeds, allied occupations, crop insurance, government schemes, etc. In earlier times, farmers got information regarding agriculture and related matters from their fellow farmers, village leaders, pamphlets etc. and along with that they preferred television and radio as source of information. However, in the present era they have started preferring new media for searching various kinds of agricultural information. That is why, the present research made an attempt to know which type of information was searched by farmers through internet. The data presented in Table 4 depicts that respondents were using internet to get agricultural information regarding weather updates (55.56\%), new technology (33.89\%), input prices and availability (13.33\%), marketing information (15.56\%) and allied occupations $(16.11 \%)$. Very few respondents searched about plant protection (2.78\%), financial assistance $(5.56 \%)$, best package of practices $(8.33 \%)$ and crop insurance $(5 \%)$. Interestingly, the large farmers accessed information in all these categories more often as compared to marginal and small farmers.

\section{Websites accessed often}

The present study found that farmers have started accessing websites for agricultural information.

It is evident form table 5 which shows that overall, nearly half of them $(47.78 \%)$ were searching agricultural information on websites from which nearly three-fourth were large farmers, 43.33 per cent were small farmers and only 31.67 per cent were marginal farmers. Mostly used websites were Krishijagran.com (13.89\%) and Krishiworld.com (12.22\%). Along with that agricoop.nic.in (2.78\%), agriquest.info (6.67\%), farmrer.gov.in and ispindia.org etc. were also accessed by farmers for information. A few farmers $(3.89 \%)$ had used YouTube and Google for searching agricultural information.

\section{Purpose of using mobile phone}

Farmers were using mobile phones for multiple purposes i.e. sending and receiving calls to relatives, family members and agricultural experts, to establish link with market, using social media etc. Table 6 shows that significant numbers of farmers $(96.67 \%)$ use the mobile for sending/receiving calls of their family members/relatives/friends whereas only 15 per cent of them were making calls to agricultural experts from which 23.33 per cent were large farmers and only 13.33 per cent and 8.33 per cent were small and large farmers, respectively. About 40.56 per cent respondents were gathering agricultural information through social media such as facebook, whattapp, YouTube etc. This indicates the fortified position that social media holds today in the dissemination of information among the farming community. Further the results show that percentages of large farmer $(48.33 \%)$ were high as compared to small (41.67\%) and marginal farmers (31.67\%). Only 11.67 per cent each were using mobile phones for establishing link with market and for seeking market information. Nearly one-fourth of the respondents $(22.78 \%)$ were using mobile phones for SMS service for updates on agriculture news.

Data showed difference between large, small and marginal farmers for accessing agricultural information. Percentages of large farmers were more for getting various types of information as compared to small and marginal farmers. 
Table.1 Distribution of farmers on the basis of their demographic profile

\begin{tabular}{|c|c|c|c|c|}
\hline Age (in Years) & $\begin{array}{l}\text { Marginal Farmers } \\
\qquad(n=60)\end{array}$ & $\begin{array}{l}\text { Small Farmers } \\
\qquad(n=60)\end{array}$ & $\begin{array}{l}\text { Large Farmers } \\
\qquad(n=60)\end{array}$ & $\begin{array}{c}\text { Total } \\
(\mathbf{N}=\mathbf{1 8 0})\end{array}$ \\
\hline $\begin{array}{l}20 \text { years } \geq \text { and } \\
<30 \text { years }\end{array}$ & $\begin{array}{c}5 \\
(8.33) \\
\end{array}$ & $\begin{array}{c}1 \\
(1.67)\end{array}$ & $\begin{array}{c}7 \\
(11.67)\end{array}$ & $\begin{array}{c}13 \\
(7.22)\end{array}$ \\
\hline $\begin{array}{l}30 \text { years } \geq \text { and } \\
<40 \text { years }\end{array}$ & $\begin{array}{c}10 \\
(16.67)\end{array}$ & $\begin{array}{c}8 \\
(13.33)\end{array}$ & $\begin{array}{c}11 \\
(18.33)\end{array}$ & $\begin{array}{c}29 \\
(16.11)\end{array}$ \\
\hline $\begin{array}{l}40 \text { years } \geq \text { and } \\
<50 \text { years }\end{array}$ & $\begin{array}{c}25 \\
(41.67)\end{array}$ & $\begin{array}{c}30 \\
(50.00)\end{array}$ & $\begin{array}{c}9 \\
(15.00)\end{array}$ & $\begin{array}{c}64 \\
(35.56)\end{array}$ \\
\hline $\begin{array}{l}50 \text { years } \geq \text { and } \\
<60 \text { years }\end{array}$ & $\begin{array}{c}15 \\
(25.00)\end{array}$ & $\begin{array}{c}11 \\
(18.33)\end{array}$ & $\begin{array}{c}23 \\
(38.83)\end{array}$ & $\begin{array}{c}49 \\
(27.22)\end{array}$ \\
\hline$\geq 60$ years & $\begin{array}{c}5 \\
(8.33)\end{array}$ & $\begin{array}{c}10 \\
(16.67)\end{array}$ & $\begin{array}{c}10 \\
(16.67)\end{array}$ & $\begin{array}{c}25 \\
(13.89)\end{array}$ \\
\hline \multicolumn{5}{|c|}{ Education Qualification } \\
\hline Illiterate & $\begin{array}{c}12 \\
(20.00)\end{array}$ & $\begin{array}{c}13 \\
(21.67)\end{array}$ & $\begin{array}{c}8 \\
(13.33)\end{array}$ & $\begin{array}{c}33 \\
(18.33)\end{array}$ \\
\hline Primary & $\begin{array}{c}6 \\
(10.00)\end{array}$ & $\begin{array}{c}7 \\
(11.67)\end{array}$ & $\begin{array}{c}6 \\
(10.00)\end{array}$ & $\begin{array}{c}19 \\
(10.56)\end{array}$ \\
\hline Middle & $\begin{array}{c}6 \\
(10.00)\end{array}$ & $\begin{array}{c}11 \\
(18.33)\end{array}$ & $\begin{array}{c}9 \\
(15.00)\end{array}$ & $\begin{array}{c}26 \\
(14.44)\end{array}$ \\
\hline Matric & $\begin{array}{c}21 \\
(35.00)\end{array}$ & $\begin{array}{c}21 \\
(35.00)\end{array}$ & $\begin{array}{c}15 \\
(25.00)\end{array}$ & $\begin{array}{c}57 \\
(31.67)\end{array}$ \\
\hline $\begin{array}{l}\text { Senior } \\
\text { Secondary }\end{array}$ & $\begin{array}{c}9 \\
(15.00)\end{array}$ & $\begin{array}{c}7 \\
(11.67)\end{array}$ & $\begin{array}{c}12 \\
(20.00)\end{array}$ & $\begin{array}{c}28 \\
(15.55)\end{array}$ \\
\hline Graduate & $\begin{array}{c}6 \\
(10.00)\end{array}$ & $\begin{array}{c}1 \\
(1.67)\end{array}$ & $\begin{array}{c}7 \\
(11.67)\end{array}$ & $\begin{array}{c}14 \\
(7.78)\end{array}$ \\
\hline $\begin{array}{l}\text { Post Graduate } \\
\text { \& above }\end{array}$ & - & - & $\begin{array}{c}3 \\
(5.00) \\
\end{array}$ & $\begin{array}{c}3 \\
(1.67) \\
\end{array}$ \\
\hline \multicolumn{5}{|l|}{ Type of Family } \\
\hline Joint & $\begin{array}{c}27 \\
(45.00)\end{array}$ & $\begin{array}{c}32 \\
(53.33)\end{array}$ & $\begin{array}{c}36 \\
(60.00)\end{array}$ & $\begin{array}{c}95 \\
(52.78)\end{array}$ \\
\hline Nuclear & $\begin{array}{c}33 \\
(55.00)\end{array}$ & $\begin{array}{c}28 \\
(46.67)\end{array}$ & $\begin{array}{c}24 \\
(40.00)\end{array}$ & $\begin{array}{c}85 \\
(47.22)\end{array}$ \\
\hline \multicolumn{5}{|c|}{ Annual Income (in Rupees) } \\
\hline$<100000$ & $\begin{array}{c}32 \\
(53.33)\end{array}$ & $\begin{array}{c}1 \\
(1.67) \\
\end{array}$ & - & $\begin{array}{c}33 \\
(18.33)\end{array}$ \\
\hline $\begin{array}{l}100000 \geq \text { and } \\
<300000\end{array}$ & $\begin{array}{c}16 \\
(26.67)\end{array}$ & $\begin{array}{c}24 \\
(40.00)\end{array}$ & $\begin{array}{c}9 \\
(15.00)\end{array}$ & $\begin{array}{c}49 \\
(27.22)\end{array}$ \\
\hline $\begin{array}{l}300000 \geq \text { and } \\
<500000\end{array}$ & $\begin{array}{c}9 \\
(15.00)\end{array}$ & $\begin{array}{c}24 \\
(40.00)\end{array}$ & $\begin{array}{c}23 \\
(38.33)\end{array}$ & $\begin{array}{c}56 \\
(31.11)\end{array}$ \\
\hline $\begin{array}{l}500000 \geq \text { and } \\
<700000\end{array}$ & $\begin{array}{c}3 \\
(5.00)\end{array}$ & $\begin{array}{c}11 \\
(18.33)\end{array}$ & $\begin{array}{c}18 \\
(30.00)\end{array}$ & $\begin{array}{c}32 \\
(17.77)\end{array}$ \\
\hline$\geq 700000$ & - & - & $\begin{array}{c}10 \\
(16.67)\end{array}$ & $\begin{array}{c}10 \\
(5.55)\end{array}$ \\
\hline
\end{tabular}

Figures in parentheses are percentages 
Table.2 Availability and use of mobile media and internet

\begin{tabular}{|c|c|c|c|c|}
\hline Particulars & $\begin{array}{c}\text { Marginal } \\
\text { Farmers } \\
(\mathbf{n}=60)\end{array}$ & $\begin{array}{l}\text { Small Farmers } \\
\qquad(\mathrm{n}=60)\end{array}$ & $\begin{array}{c}\text { Large Farmers } \\
\qquad(\mathrm{n}=60)\end{array}$ & $\begin{array}{c}\text { Total } \\
(\mathbf{N}=\mathbf{1 8 0})\end{array}$ \\
\hline \multicolumn{5}{|c|}{ Access to Mobile Phones } \\
\hline Yes & $\begin{array}{c}56 \\
(93.33)\end{array}$ & $\begin{array}{c}60 \\
(100.00)\end{array}$ & $\begin{array}{c}59 \\
(98.33)\end{array}$ & $\begin{array}{c}175 \\
(97.22)\end{array}$ \\
\hline No & $\begin{array}{c}4 \\
(6.67)\end{array}$ & - & $\begin{array}{c}1 \\
(1.67)\end{array}$ & $\begin{array}{c}5 \\
(2.78)\end{array}$ \\
\hline \multicolumn{5}{|c|}{ Number of Mobiles are there at home } \\
\hline None & $\begin{array}{c}3 \\
(5.00)\end{array}$ & - & - & $\begin{array}{c}3 \\
(1.67)\end{array}$ \\
\hline One & $\begin{array}{c}23 \\
(38.33)\end{array}$ & $\begin{array}{c}18 \\
(30.00)\end{array}$ & $\begin{array}{c}9 \\
(15.00)\end{array}$ & $\begin{array}{c}50 \\
(27.78)\end{array}$ \\
\hline Two & $\begin{array}{c}25 \\
(41.67)\end{array}$ & $\begin{array}{c}34 \\
(56.67)\end{array}$ & $\begin{array}{c}28 \\
(46.67)\end{array}$ & $\begin{array}{c}87 \\
(48.33)\end{array}$ \\
\hline Three or more & $\begin{array}{c}9 \\
(15.00)\end{array}$ & $\begin{array}{c}8 \\
(13.33)\end{array}$ & $\begin{array}{c}23 \\
(38.33)\end{array}$ & $\begin{array}{c}40 \\
(22.22)\end{array}$ \\
\hline \multicolumn{5}{|c|}{ Type of Mobile available at home } \\
\hline $\begin{array}{l}\text { Only Ordinary } \\
\text { Phone }\end{array}$ & $\begin{array}{c}30 \\
(50.00)\end{array}$ & $\begin{array}{c}24 \\
(40.00)\end{array}$ & $\begin{array}{c}13 \\
(21.67)\end{array}$ & $\begin{array}{c}67 \\
(37.22)\end{array}$ \\
\hline $\begin{array}{l}\text { Only Smart } \\
\text { Phone }\end{array}$ & $\begin{array}{c}21 \\
(35.00)\end{array}$ & $\begin{array}{c}26 \\
(43.33)\end{array}$ & $\begin{array}{c}25 \\
(41.67)\end{array}$ & $\begin{array}{c}71 \\
(40.00)\end{array}$ \\
\hline $\begin{array}{l}\text { Ordinary Phone + } \\
\text { Smart Phone }\end{array}$ & $\begin{array}{c}9 \\
(15.00)\end{array}$ & $\begin{array}{c}10 \\
(16.67)\end{array}$ & $\begin{array}{c}22 \\
(36.67)\end{array}$ & $\begin{array}{c}41 \\
(22.78)\end{array}$ \\
\hline \multicolumn{5}{|c|}{ Availability of internet connection on Phones } \\
\hline Yes & $\begin{array}{c}30 \\
(50.00)\end{array}$ & $\begin{array}{c}36 \\
(60.00)\end{array}$ & $\begin{array}{c}47 \\
(78.33)\end{array}$ & $\begin{array}{c}113 \\
(62.78)\end{array}$ \\
\hline IT & $\begin{array}{c}30 \\
(50.00)\end{array}$ & $\begin{array}{c}24 \\
(40.00)\end{array}$ & $\begin{array}{c}13 \\
(21.67)\end{array}$ & $\begin{array}{c}67 \\
(37.22)\end{array}$ \\
\hline \multicolumn{5}{|c|}{ Hours spent on mobile phone in a day } \\
\hline No use & $\begin{array}{c}9 \\
(15.00)\end{array}$ & $\begin{array}{c}5 \\
(8.33)\end{array}$ & $\begin{array}{c}1 \\
(1.67)\end{array}$ & $\begin{array}{c}15 \\
(8.33)\end{array}$ \\
\hline$<1$ & $\begin{array}{c}31 \\
(51.67)\end{array}$ & $\begin{array}{c}31 \\
(51.67)\end{array}$ & $\begin{array}{c}17 \\
(28.33)\end{array}$ & $\begin{array}{c}79 \\
(43.89)\end{array}$ \\
\hline $1-2$ & $\begin{array}{c}13 \\
(21.67)\end{array}$ & $\begin{array}{c}14 \\
(23.33)\end{array}$ & $\begin{array}{c}23 \\
(38.33)\end{array}$ & $\begin{array}{c}50 \\
(27.78)\end{array}$ \\
\hline $2-3$ & $\begin{array}{c}6 \\
(10.00)\end{array}$ & $\begin{array}{c}8 \\
(13.33)\end{array}$ & $\begin{array}{c}15 \\
(8.33)\end{array}$ & $\begin{array}{c}29 \\
(16.11)\end{array}$ \\
\hline 3-4 & $\begin{array}{c}1 \\
(1.67)\end{array}$ & $\begin{array}{c}2 \\
(3.33)\end{array}$ & $\begin{array}{c}4 \\
(6.67)\end{array}$ & $\begin{array}{c}7 \\
(3.89)\end{array}$ \\
\hline
\end{tabular}

Figures in parentheses are percentages 
Table.3 Need of assistance by family members while operating online medium

\begin{tabular}{|l|c|c|c|c|}
\hline & $\begin{array}{c}\text { Marginal Farmers } \\
(\mathbf{n = 6 0})\end{array}$ & $\begin{array}{c}\text { Small Farmers } \\
(\mathbf{n = 6 0})\end{array}$ & $\begin{array}{c}\text { Large Farmers } \\
(\mathbf{n = 6 0})\end{array}$ & $\begin{array}{c}\text { Total } \\
(\mathbf{N = 1 8 0})\end{array}$ \\
\hline Yes & 29 & 27 & 32 & 88 \\
& $(48.33)$ & $(45.00)$ & $(53.33)$ & $(48.89)$ \\
\hline No & 31 & 33 & 28 & 92 \\
& $(51.67)$ & $(55.00)$ & $(46.67)$ & $(51.11)$ \\
\hline
\end{tabular}

Figures in parentheses are percentages

Table.4 Type of agricultural information searched through internet

*Multiple Responses

\begin{tabular}{|c|c|c|c|c|}
\hline & $\begin{array}{l}\text { Marginal Farmers } \\
\qquad(\mathrm{n}=60)\end{array}$ & $\begin{array}{l}\text { Small Farmers } \\
\qquad(\mathrm{n}=60)\end{array}$ & $\begin{array}{l}\text { Large Farmers } \\
\qquad(n=60)\end{array}$ & $\begin{array}{c}\text { Total } \\
(\mathrm{N}=\mathbf{1 8 0})\end{array}$ \\
\hline Weather report & $\begin{array}{c}26 \\
(43.33)\end{array}$ & $\begin{array}{c}34 \\
(56.67)\end{array}$ & $\begin{array}{c}40 \\
(66.67)\end{array}$ & $\begin{array}{c}100 \\
(55.56)\end{array}$ \\
\hline New technology & $\begin{array}{c}11 \\
(18.33)\end{array}$ & $\begin{array}{c}18 \\
(30.00)\end{array}$ & $\begin{array}{c}32 \\
(53.33)\end{array}$ & $\begin{array}{c}61 \\
(33.89)\end{array}$ \\
\hline $\begin{array}{l}\text { Input prices and } \\
\text { availability }\end{array}$ & $\begin{array}{c}3 \\
(5.00)\end{array}$ & $\begin{array}{c}6 \\
(10.00)\end{array}$ & $\begin{array}{c}15 \\
(25.00)\end{array}$ & $\begin{array}{c}24 \\
(13.33)\end{array}$ \\
\hline $\begin{array}{l}\text { Marketing } \\
\text { information }\end{array}$ & $\begin{array}{c}6 \\
(10.00)\end{array}$ & $\begin{array}{c}8 \\
(13.33)\end{array}$ & $\begin{array}{c}14 \\
(23.33)\end{array}$ & $\begin{array}{c}28 \\
(15.56)\end{array}$ \\
\hline $\begin{array}{l}\text { Plant protection } \\
\text { technology }\end{array}$ & $\begin{array}{c}2 \\
(3.33)\end{array}$ & $\begin{array}{c}1 \\
(1.67)\end{array}$ & $\begin{array}{c}2 \\
(3.33)\end{array}$ & $\begin{array}{c}5 \\
(2.78)\end{array}$ \\
\hline $\begin{array}{l}\text { Financial } \\
\text { assistance/Bank } \\
\text { loans }\end{array}$ & $\begin{array}{c}3 \\
(5.00)\end{array}$ & $\begin{array}{c}2 \\
(3.33)\end{array}$ & $\begin{array}{c}5 \\
(8.33)\end{array}$ & $\begin{array}{c}10 \\
(5.56)\end{array}$ \\
\hline $\begin{array}{l}\text { Best package of } \\
\text { practices }\end{array}$ & $\begin{array}{c}5 \\
(8.33)\end{array}$ & $\begin{array}{c}3 \\
(5.00)\end{array}$ & $\begin{array}{c}7 \\
(11.67)\end{array}$ & $\begin{array}{c}15 \\
(8.33)\end{array}$ \\
\hline Crop Insurance & $\begin{array}{c}2 \\
(3.33)\end{array}$ & $\begin{array}{c}3 \\
(5.00)\end{array}$ & $\begin{array}{c}4 \\
(6.67)\end{array}$ & $\begin{array}{c}9 \\
(5.00)\end{array}$ \\
\hline Allied occupations & $\begin{array}{c}4 \\
(6.67)\end{array}$ & $\begin{array}{c}9 \\
(15.00)\end{array}$ & $\begin{array}{c}16 \\
(26.67)\end{array}$ & $\begin{array}{c}29 \\
(16.11)\end{array}$ \\
\hline
\end{tabular}

Figures in parentheses are percentages 
Table.5 Website accessed by farmers often

**Multiple Responses

\begin{tabular}{|c|c|c|c|c|}
\hline Websites & $\begin{array}{l}\text { Marginal Farmers } \\
\qquad(\mathrm{n}=60)\end{array}$ & $\begin{array}{l}\text { Small Farmers } \\
\qquad(\mathrm{n}=60)\end{array}$ & $\begin{array}{l}\text { Large Farmers } \\
\qquad(\mathbf{n}=60)\end{array}$ & $\begin{array}{c}\text { Total } \\
(\mathrm{N}=180)\end{array}$ \\
\hline agricoop.nic.in & - & $\begin{array}{c}1 \\
(1.67)\end{array}$ & $\begin{array}{c}4 \\
(6.67)\end{array}$ & $\begin{array}{c}5 \\
(2.78)\end{array}$ \\
\hline agriquest.info & $\begin{array}{c}4 \\
(6.67)\end{array}$ & $\begin{array}{c}2 \\
(3.33)\end{array}$ & $\begin{array}{c}6 \\
(10.00)\end{array}$ & $\begin{array}{c}12 \\
(6.67)\end{array}$ \\
\hline agriwatch.com & $\begin{array}{c}3 \\
(5.00)\end{array}$ & $\begin{array}{c}4 \\
(6.67)\end{array}$ & $\begin{array}{c}1 \\
(1.67)\end{array}$ & $\begin{array}{c}8 \\
(4.44)\end{array}$ \\
\hline farmer.gov.in & - & $\begin{array}{c}1 \\
(1.67)\end{array}$ & $\begin{array}{c}2 \\
(3.33)\end{array}$ & $\begin{array}{c}3 \\
(1.67)\end{array}$ \\
\hline isapindia.org & - & - & $\begin{array}{c}3 \\
(5.00)\end{array}$ & $\begin{array}{c}3 \\
(1.67)\end{array}$ \\
\hline rmlglobal.com & $\begin{array}{c}1 \\
(1.67)\end{array}$ & - & - & $\begin{array}{c}1 \\
(0.56)\end{array}$ \\
\hline krishijagran.com & $\begin{array}{c}5 \\
(8.33)\end{array}$ & $\begin{array}{c}7 \\
(11.67)\end{array}$ & $\begin{array}{c}13 \\
(21.67)\end{array}$ & $\begin{array}{c}25 \\
(13.89)\end{array}$ \\
\hline agmarknet.nic.in & $\begin{array}{c}4 \\
(6.67)\end{array}$ & $\begin{array}{c}1 \\
(1.67)\end{array}$ & $\begin{array}{c}2 \\
(3.33)\end{array}$ & $\begin{array}{c}7 \\
(3.89)\end{array}$ \\
\hline fert.nic.in & - & - & $\begin{array}{c}1 \\
(1.67)\end{array}$ & $\begin{array}{c}1 \\
(0.56)\end{array}$ \\
\hline krishiworld.com & $\begin{array}{c}2 \\
(3.33)\end{array}$ & $\begin{array}{c}9 \\
(15.00)\end{array}$ & $\begin{array}{c}11 \\
(18.33)\end{array}$ & $\begin{array}{c}22 \\
(12.22)\end{array}$ \\
\hline indiaagristat.com & - & $\begin{array}{c}1 \\
(1.67)\end{array}$ & - & $\begin{array}{c}1 \\
(0.56)\end{array}$ \\
\hline fciweb.nic.in & - & - & $\begin{array}{c}1 \\
(1.67)\end{array}$ & $\begin{array}{c}1 \\
(0.56)\end{array}$ \\
\hline Others* & - & $\begin{array}{c}6 \\
(10.00)\end{array}$ & $\begin{array}{c}1 \\
(1.67)\end{array}$ & $\begin{array}{c}7 \\
(3.89)\end{array}$ \\
\hline Total & $\begin{array}{c}19 \\
(31.67)\end{array}$ & $\begin{array}{c}26 \\
(43.33)\end{array}$ & $\begin{array}{c}45 \\
(75.00)\end{array}$ & $\begin{array}{c}90 \\
(50.00)\end{array}$ \\
\hline
\end{tabular}

Figures in parentheses are percentages

*You Tube and Google 
Table.6 Main Purpose of using mobile phone

*Multiple Responses

\begin{tabular}{|c|c|c|c|c|}
\hline & $\begin{array}{l}\text { Marginal Farmers } \\
\qquad(\mathrm{n}=60)\end{array}$ & $\begin{array}{c}\text { Small Farmers } \\
\quad(n=60)\end{array}$ & $\begin{array}{l}\text { Large Farmers } \\
\quad(n=60)\end{array}$ & $\begin{array}{c}\text { Total } \\
(\mathbf{N}=\mathbf{1 8 0})\end{array}$ \\
\hline $\begin{array}{l}\text { Sending/receiving calls to } \\
\text { friends/family/relatives }\end{array}$ & $\begin{array}{c}58 \\
(96.67)\end{array}$ & $\begin{array}{c}57 \\
(95.00)\end{array}$ & $\begin{array}{c}59 \\
(98.33)\end{array}$ & $\begin{array}{c}174 \\
(96.67)\end{array}$ \\
\hline $\begin{array}{l}\text { Sending/receiving calls to } \\
\text { agriculture experts }\end{array}$ & $\begin{array}{c}5 \\
(8.33)\end{array}$ & $\begin{array}{c}8 \\
(13.33)\end{array}$ & $\begin{array}{c}14 \\
(23.33)\end{array}$ & $\begin{array}{c}27 \\
(15.00)\end{array}$ \\
\hline $\begin{array}{l}\text { Access to extension } \\
\text { services }\end{array}$ & $\begin{array}{c}2 \\
(3.33)\end{array}$ & $\begin{array}{c}9 \\
(15.00)\end{array}$ & $\begin{array}{c}3 \\
(5.00)\end{array}$ & $\begin{array}{c}9 \\
(5.00)\end{array}$ \\
\hline $\begin{array}{l}\text { Establishing market } \\
\text { links }\end{array}$ & $\begin{array}{c}1 \\
(1.67)\end{array}$ & $\begin{array}{c}5 \\
(8.33)\end{array}$ & $\begin{array}{c}15 \\
(25.00)\end{array}$ & $\begin{array}{c}21 \\
(11.67)\end{array}$ \\
\hline $\begin{array}{l}\text { Seeking market } \\
\text { information }\end{array}$ & $\begin{array}{c}1 \\
(1.67)\end{array}$ & $\begin{array}{c}6 \\
(10.00)\end{array}$ & 12 & $\begin{array}{c}21 \\
(11.67)\end{array}$ \\
\hline $\begin{array}{l}\text { Gathering agriculture } \\
\text { related information } \\
\text { through social media like } \\
\text { Facebook, whatsapp, } \\
\text { Youtube etc. }\end{array}$ & $\begin{array}{c}19 \\
(31.67)\end{array}$ & $\begin{array}{c}25 \\
(41.67)\end{array}$ & $\begin{array}{c}29 \\
(48.33)\end{array}$ & $\begin{array}{c}73 \\
(40.56)\end{array}$ \\
\hline $\begin{array}{l}\text { Using SMS service for } \\
\text { updates on agriculture } \\
\text { news }\end{array}$ & $\begin{array}{c}10 \\
(16.67)\end{array}$ & $\begin{array}{c}12 \\
(20.00)\end{array}$ & $\begin{array}{c}19 \\
(31.67)\end{array}$ & $\begin{array}{c}41 \\
(22.78)\end{array}$ \\
\hline Listening to music & $\begin{array}{c}13 \\
(21.67)\end{array}$ & $\begin{array}{c}16 \\
(26.67)\end{array}$ & $\begin{array}{c}23 \\
(38.33)\end{array}$ & $\begin{array}{c}52 \\
(28.89)\end{array}$ \\
\hline Chat & $\begin{array}{c}18 \\
(30.00)\end{array}$ & $\begin{array}{c}23 \\
(38.33)\end{array}$ & $\begin{array}{c}26 \\
(43.33)\end{array}$ & $\begin{array}{c}67 \\
(37.22)\end{array}$ \\
\hline $\begin{array}{l}\text { Watching films/songs } \\
\text { through mobiles internet }\end{array}$ & $\begin{array}{c}13 \\
(21.67) \\
\end{array}$ & $\begin{array}{c}15 \\
(25.00)\end{array}$ & $\begin{array}{c}23 \\
(38.33) \\
\end{array}$ & $\begin{array}{c}51 \\
(28.33) \\
\end{array}$ \\
\hline Weather updates & $\begin{array}{c}15 \\
(25.00)\end{array}$ & $\begin{array}{c}30 \\
(50.00)\end{array}$ & $\begin{array}{c}30 \\
(50.00)\end{array}$ & $\begin{array}{c}75 \\
(41.67)\end{array}$ \\
\hline
\end{tabular}

Figures in parentheses are percentages 
Table.7 Distribution of respondents according to the use of mobile based Agri. Apps for getting agricultural information

\begin{tabular}{|c|c|c|c|c|}
\hline & $\begin{array}{l}\text { Marginal Farmers } \\
\qquad(n=60)\end{array}$ & $\begin{array}{l}\text { Small Farmers } \\
\qquad(n=60)\end{array}$ & $\begin{array}{l}\text { Large Farmers } \\
\qquad(n=60)\end{array}$ & $\begin{array}{c}\text { Total } \\
(\mathbf{N}=\mathbf{1 8 0})\end{array}$ \\
\hline Yes & $\begin{array}{c}13 \\
(21.67)\end{array}$ & $\begin{array}{c}23 \\
(38.33)\end{array}$ & $\begin{array}{c}30 \\
(50.00)\end{array}$ & $\begin{array}{c}66 \\
(36.67)\end{array}$ \\
\hline $\begin{array}{l}\text { If yes, Which mobile } \\
\text { based agro-advisory } \\
\text { service or agri apps } \\
\text { do you use }\end{array}$ & $\begin{array}{l}\text { Marginal Farmers } \\
\qquad\left(n_{1}=13\right)\end{array}$ & $\begin{array}{l}\text { Small Farmers } \\
\qquad\left(\mathrm{n}_{2}=23\right)\end{array}$ & $\begin{array}{l}\text { Large Farmers } \\
\qquad\left(\mathbf{n}_{3}=\mathbf{3 0}\right)\end{array}$ & $\begin{array}{c}\text { Total } \\
(n=66)\end{array}$ \\
\hline \multicolumn{5}{|c|}{ *Multiple Response } \\
\hline IFFCO Kisan & $\begin{array}{c}10 \\
(76.92)\end{array}$ & $\begin{array}{c}11 \\
(47.83)\end{array}$ & $\begin{array}{c}17 \\
(56.67)\end{array}$ & $\begin{array}{c}38 \\
(57.58)\end{array}$ \\
\hline KisanSuvidha & $\begin{array}{c}2 \\
(15.38)\end{array}$ & $\begin{array}{c}5 \\
(21.74)\end{array}$ & $\begin{array}{c}10 \\
(33.33)\end{array}$ & $\begin{array}{c}17 \\
(25.76)\end{array}$ \\
\hline Kheti-Badi & $\begin{array}{c}1 \\
(7.69)\end{array}$ & $\begin{array}{c}6 \\
(26.09)\end{array}$ & $\begin{array}{c}5 \\
(16.67)\end{array}$ & $\begin{array}{c}12 \\
(18.18)\end{array}$ \\
\hline AgriApp & - & 3 & $\begin{array}{c}7 \\
(23.33)\end{array}$ & $\begin{array}{c}10 \\
(15.15)\end{array}$ \\
\hline Fertilizer calculator & $\begin{array}{c}1 \\
(7.69)\end{array}$ & - & $\begin{array}{c}2 \\
(6.67)\end{array}$ & $\begin{array}{c}3 \\
(4.55)\end{array}$ \\
\hline PusaKrishi & $\begin{array}{c}2 \\
(15.38)\end{array}$ & $\begin{array}{c}6 \\
(26.09)\end{array}$ & $\begin{array}{c}7 \\
(23.33)\end{array}$ & $\begin{array}{c}15 \\
(22.73)\end{array}$ \\
\hline KrishiGyan & $\begin{array}{c}3 \\
(23.08)\end{array}$ & $\begin{array}{c}10 \\
(43.48)\end{array}$ & $\begin{array}{c}18 \\
(60.00)\end{array}$ & $\begin{array}{c}31 \\
(46.97)\end{array}$ \\
\hline Crop Insurance & - & $\begin{array}{c}1 \\
(4.35)\end{array}$ & - & $\begin{array}{c}1 \\
(1.52)\end{array}$ \\
\hline AgriMarket & - & - & $\begin{array}{c}2 \\
(6.67)\end{array}$ & $\begin{array}{c}2 \\
(3.03)\end{array}$ \\
\hline $\begin{array}{lr}\text { Criteria of } & \text { selecting } \\
\text { the apps } & \text { for } \\
\text { agricultural } & \\
\text { information } & \text { by } \\
\text { farmers }\end{array}$ & $\begin{array}{l}\text { Marginal Farmers } \\
\qquad\left(n_{1}=13\right)\end{array}$ & $\begin{array}{l}\text { Small Farmers } \\
\qquad\left(\mathrm{n}_{2}=23\right)\end{array}$ & $\begin{array}{l}\text { Large Farmers } \\
\qquad\left(\mathbf{n}_{3}=\mathbf{3 0}\right)\end{array}$ & $\begin{array}{c}\text { Total } \\
(n=66)\end{array}$ \\
\hline Expert advice & $\begin{array}{c}2 \\
(15.38)\end{array}$ & $\begin{array}{c}14 \\
(60.87)\end{array}$ & $\begin{array}{c}20 \\
(66.67)\end{array}$ & $\begin{array}{c}36 \\
(54.55)\end{array}$ \\
\hline Popularity & $\begin{array}{c}3 \\
(23.08)\end{array}$ & $\begin{array}{c}2 \\
(8.70)\end{array}$ & $\begin{array}{c}1 \\
(3.33)\end{array}$ & $\begin{array}{c}5 \\
(7.58)\end{array}$ \\
\hline Choose randomly & $\begin{array}{c}1 \\
(7.69)\end{array}$ & $\begin{array}{c}3 \\
(13.04)\end{array}$ & $\begin{array}{c}4 \\
(13.33)\end{array}$ & $\begin{array}{c}8 \\
(12.12)\end{array}$ \\
\hline $\begin{array}{l}\text { Suggested by } \\
\text { friends/relatives }\end{array}$ & $\begin{array}{c}7 \\
(53.85)\end{array}$ & $\begin{array}{c}4 \\
(17.39)\end{array}$ & $\begin{array}{c}5 \\
(16.67)\end{array}$ & $\begin{array}{c}16 \\
(8.89)\end{array}$ \\
\hline
\end{tabular}

Figures in parentheses are percentages 
Table.8 Subscribed to online agri news through whatsapp like Kheti Sandesh

\begin{tabular}{|l|c|c|c|c|}
\hline & $\begin{array}{c}\text { Marginal Farmers } \\
(\mathbf{n = 6 0})\end{array}$ & $\begin{array}{c}\text { Small Farmers } \\
(\mathbf{n = 6 0})\end{array}$ & $\begin{array}{c}\text { Large Farmers } \\
(\mathbf{n = 6 0})\end{array}$ & $\begin{array}{c}\text { Total } \\
(\mathbf{N}=\mathbf{1 8 0})\end{array}$ \\
\hline Yes & 10 & 18 & 25 & 53 \\
& $(16.67)$ & $(30.00)$ & $(41.67)$ & $(29.44)$ \\
\hline No & 50 & 42 & 35 & 127 \\
& $(83.33)$ & $(70.00)$ & $(58.33)$ & $(70.56)$ \\
\hline
\end{tabular}

Figures in parentheses are percentages

Table.9 Perception of farmers regarding usefulness of online information

\begin{tabular}{|l|c|c|c|c|}
\hline & $\begin{array}{c}\text { Marginal Farmers } \\
(\mathbf{n = 6 0})\end{array}$ & $\begin{array}{c}\text { Small Farmers } \\
(\mathbf{n = 6 0})\end{array}$ & $\begin{array}{c}\text { Large Farmers } \\
(\mathbf{n = 6 0})\end{array}$ & $\begin{array}{c}\text { Total } \\
(\mathbf{N = 1 8 0})\end{array}$ \\
\hline Yes & 18 & 25 & 30 & 73 \\
& $(30.00)$ & $(41.67)$ & $(50.00)$ & $(40.56)$ \\
\hline No & 17 & 14 & 14 & 45 \\
& $(28.33)$ & $(23.33)$ & $(23.33)$ & $(25.00)$ \\
\hline Can't say & 25 & 21 & 16 & 62 \\
& $(41.67)$ & $(35.00)$ & $(26.67)$ & $(34.44)$ \\
\hline
\end{tabular}

Figures in parentheses are percentages

Table.10 Problems faced by farmers in accessing new media

\begin{tabular}{|l|c|c|c|c|}
\hline Problems & $\begin{array}{c}\text { Marginal Farmers } \\
(\mathbf{n = 6 0})\end{array}$ & $\begin{array}{c}\text { Small Farmers } \\
(\mathbf{n = 6 0})\end{array}$ & $\begin{array}{c}\text { Large Farmers } \\
(\mathbf{n = 6 0})\end{array}$ & $\begin{array}{c}\text { Total } \\
(\mathbf{N = 1 8 0})\end{array}$ \\
\hline $\begin{array}{l}\text { Language } \\
\text { Barrier }\end{array}$ & 44 & 37 & 32 & 113 \\
\hline Time Constraint & $(73.33)$ & $(61.67)$ & $(53.33)$ & $(62.78)$ \\
\hline Lack of & 12 & 7 & 10 & 29 \\
awareness & $(20.00)$ & $(11.67)$ & $(16.67)$ & $(16.11)$ \\
\hline $\begin{array}{l}\text { Information too } \\
\text { old /not latest }\end{array}$ & 31 & 29 & 25 & 85 \\
\hline $\begin{array}{l}\text { Information not } \\
\text { easily accessible }\end{array}$ & $(51.67)$ & $(48.33)$ & $(41.67)$ & $(47.22)$ \\
\hline $\begin{array}{l}\text { Use of } \\
\text { technical/difficult } \\
\text { words }\end{array}$ & $(10$ & 9 & 16 & 35 \\
\hline
\end{tabular}

Figures in parentheses are percentages

Usage of mobile based agri. apps

Out of total respondents, only 36.67 per cent were using mobile based Agri. Apps for getting agricultural information from which half of the large farmers accessed Agri. Apps as compared to small (38.33\%) and marginal farmers (21.67\%). Further, table 7 explains the types of 
agro-advisory service or Agri. Apps used by respondents. It was found that the most of the farmers used IFFFCO Kisan app (57.58\%), Kisan Suvidha (25.76\%), Kheti Badi (18.18\%), Kirshi Gyan (46.97\%), Pusa Krishi (22.73\%).

Results showed that IFFCO Kisan and Krishi Gyan apps were the most often used by many farmers who used Agri. Apps. When these farmers were asked about the criteria of selecting Agri. Apps, 54.55 per cent users relied on expert advice while 12.12 per cent chose them randomly. Another 8.89 per cent were suggested by friends and relatives where as 7.58 per cent chose them on the basis of their popularity. This shows that the farmers rely heavily on experts for selection of Agri. Apps.

\section{Subscribed to online agri news through whatsapp like Kheti Sandesh}

Punjab Agricultural University has started an app which connects farmers through whattapp for regular agricultural updates i.e. Kheti Sandesh. The perusal of table 8 indicates that only 29.44 per cent of farmers were having subscription of online agri news through whattapp like Kheti Sandesh whereas 70.56 per cent had not subscribed to it as they were not aware of such an app or the procedure for subscription. It means news subscription through whattsapp is yet to catch the fancy of a majority of farmers and the organizations offering them will have to create awareness about them.

\section{Perception of farmers regarding usefulness of online information}

The data presented in table 9 explains the perception of farmers regarding usefulness of online information of agriculture. They were asked whether agricultural information, they had acquired through online medium, was really helpful for them or not. Only 40.56 per cent farmers reported that online agriculture information was really useful whereas one fourth of them had showed a negative attitude regarding usefulness of online information and 34.44 per cent of them had neutral approach.

\section{Problems}

New media has provided multidimensional benefits to farmers. In spite of that, farmers have to face many constraints while operating new media. So the study found that major problem faced by farmers $(62.78 \%)$ was language barrier as they were not able to understand the language on apps and on websites, from which marginal farmers, due to illiteracy or less education, had highest percentages i.e. 73.33 per cent as compared to small $(61.67 \%)$ and large farmers $(53.33 \%)$. This is due to the reason that most of Mapps/websites are in English language. The study is also in line with the findings of Syiem and Raj (2016), Jain et al., (2015), Kaur et al., (2013), Shankariah and Swamy (2012) and Olaniyi (2012). Though, some farmers were able to understand language but they were incapable to comprehend the exact meaning of technical/ difficult words used in apps/websites $(58.89 \%)$, from which again this problem was faced more by marginal farmers $(63.33 \%)$ as compared to small $(63.35 \%)$ and large farmers $(53.33 \%)$. (Table 10)

Nearly half of the farmers $(47.22 \%)$ pointed out that there was lack of awareness, training and practical exposure viz-a-viz new media and they felt that there was a need of training about how to use M-Apps/websites for searching agriculture related information. This corroborates the study conducted by Syiem and Raj (2016) who reported same findings regarding lack of skill in handling ICTs, training and exposure. Along with that, some farmers $(19.44 \%)$ reported that information available on apps and websites was either old or not up to date.

Conclusion and Recommendations of the study are as follows:

It can be concluded from the study that farmers have started using new media as the easiest and 
cheapest way to access information or knowledge about weather news/updates, new technology, government schemes, bank loans, etc. However, the study has found that lack of awareness about using new media, language problem, impractical and old information etc were constraints faced by farmers. Since there is increasing penetration of devices like mobile phones and computers due to affordability of new media among farmers of the state, there is a need to ensure that their problems are being addressed. This would enable the farming community derive maximum benefits by way of better access to information services through use of new media for agriculture and other development processes. Based on the results of the study, the following suggestions are given:

1. Information available on apps and websites should be regularly updated and it should be practical in nature.

2. Farmers should be made aware about information through new media by massive awareness drives by Government.

3. Avoidance of technical jargon and use of vernacular language in new media should be promoted.

4. Co-operative societies should be roped in to create online and whatsapp groups to provide information to member farmers.

5. User interface should be simplified so that farmers can easily access the agricultural information.

\section{References}

Anonymous (2012) State Bank of India Agriculture report. Retrieved from: http://agricoop.nic.in/SIA111213312.pdf on 28 March, 2018.
Bachhav, N B (2012) Information Needs of the Rural Farmers: A Study from Maharashtra, India: A Survey. Library Philosophy and Practice (e-journal) http://digitalcommons.unl.edu/libphilprac/ 866

Chhachhar A R, Querestic B, Khusk G M, and Ahmed $S$ (2014) Impact of ICTs in Agriculture Development, J. of Basic Applied Scientific Research 4(1):281288.

Herzberg A (2003) Payments and banking with mobile personal devices. Comm ACM 46: $53-58$.

Olaniyi O A (2013) Assessment of Utilization of Information and Communication Technologies (ICTs) among Poultry Farmers in Nigeria: An Emerging Challenge. $\mathrm{J}$ of Animal Sciences Advances 3(7):361-369.

Shankaraiah N and Swamy B K N (2012) Mobile Communication as a Viable Tool for Agriculture and Rural Development, Proceedings of Mobile for development held on 2012. Department of Agricultural Extension, University of Agricultural Sciences, Bangalore.

Syiem R and Raj S (2015) Access and usage of ICTs for agricultural and rural development by the tribal farmers in Meghalaya state of north-east India. J. of Agri. Informatics 6(3):24-41.

Thapar S and Nayal A (2018) Online and Mobile Media Utilization among the Farmers of Punjab- Some Opportunities and Challenges. International J. of New Media Studies 7 (4): 41-46.

Yuen A H K, Law N, Wong K C (2003) ICT implementation and school leadership: Case studies of ICT integration in teaching and learning. J Edu Adminis 41: $158-70$.

\section{How to cite this article:}

Sheetal Thapar, Narinderpal Singh and Ranjeet Kaur. 2019. Utilization of New Media among Farmers of Punjab: A Study of Moga District, India. Int.J.Curr.Microbiol.App.Sci. 8(10): 23442357. doi: https://doi.org/10.20546/ijcmas.2019.810.271 